\title{
Advances in opportunistic radio technologies for TVWS
}

\author{
Dominique Noguet, Matthieu Gautier and Vincent Berg
}

\begin{abstract}
Cognitive radio has been an active research area in wireless communications over the past 10 years. TV Digital Switch Over resulted in new regulatory regimes, which offer the first large-scale opportunity for cognitive radio and networks. This article considers the most recent regulatory rules for TV White Space opportunistic usage, and proposes technologies to operate in these bands. It addresses techniques to assess channel vacancy by the cognitive radio, focusing on the two incumbent systems of the TV bands, namely TV stations and wireless microphones. Spectrum-sensing performance is discussed under TV White Space regulation parameters. Then, modulation schemes for the opportunistic radio are discussed, showing the limitations of classical multi-carrier techniques and the advantages of filter bank modulations. In particular, the low adjacent band leakage of filter bank is addressed, and its benefit for spectrum pooling is stressed as a means to offer broadband access through channel aggregation.
\end{abstract}

\section{Introduction}

Cognitive radio (CR) has been a major research topic in the radio communication community since it was introduced by Mitola in the early 2000s [1]. Cognitive Radio capitalizes on the flexible radio concept by setting the configuration of a flexible radio according to sensing and decision-making capabilities. Unfortunately, largescale deployment of CR in civil telecommunication systems was somewhat limited. This is mainly because its full benefits would require disruptive regulation rules, where spectrum resources would be allocated according to actual needs at a specific location in space and time, rather than ruled by fixed frequency assignments [2]. Therefore, first experimental over-the-air testbeds were restricted to the ISM bands [3-5] and/or to the research community [6,7].

In some countries, the so-called digital switch over (DSO) in TV bands has recently resulted in relaxed regulatory regimes, where unlicensed spectrum use is allowed, provided that non-harmful interference to incumbent systems is guaranteed. This move was first initiated in the USA, where the first proposed rules for the "Unlicensed Operation in the TV Broadcast Bands" were published as early as 2004 [8], with the final set of

\footnotetext{
* Correspondence: dominique.noguet@cea.fr

CEA-LETI MINATEC, 17 rue des Martyrs, 38054 Grenoble Cedex 9, France
}

rules in 2009 [9] and an additional notice in 2011 [10]. A similar trend is currently under way in other countries: for example, in the UK [11], Europe [12], and Australia [13]. As a consequence, the unused TV spectrum, referred to as TV White Space (TVWS), has become one of the very first portions of the spectrum where the concepts of CR meet regulatory and actual deploymentdriven requirement figures.

Standardization actions to exploit the TVWS have been carried out within IEEE802 groups, first in IEEE802.22 [14], and more recently in IEEE802.11af [15]. Both standards further develop previous technologies (respectively, IEEE802.16 and IEEE802.11a) to make them fit the specific requirements of the TVWS to enable rapid availability of white space devices (WSD) on the market. Similarly, the study of the CogNea consortium to deliver the ECMA392 standard can also be mentioned [16,17].

This article aims at highlighting the challenges to improve TVWS resource usage, suggesting new physical layer (PHY) options compared to those developed in these standards. Section 2 summarizes the regulation regimes for TV spectrum in the USA, the UK, and other countries to identify the key technical requirements for the rest of the article. Section 3 describes techniques to assess the vacancy of TV channels and ensure noninterference with incumbents. The detection of both 
digital terrestrial television (DTT) and wireless microphones is discussed, considering sensing and geo-location based approaches. Section 4 analyzes the specific nature of the fragmented TVWS and the stringent requirements on adjacent band leakage to suggest a new approach for the PHY in the TVWS, using filter bank modulation (FBMC) and spectrum pooling techniques.

\section{Context of the TVWS and key requirements}

The DSO has pushed regulators to restack TV channel allocation, thanks to the higher spectrum efficiency of DTT over its analog counterpart. This resulted in "cleared spectrum" portions also called "the digital dividend." In addition, for spectrum planning reasons, the channels allocated to TV transmitters are interleaved, and only few channels are used by TV broadcasts at one specific location [18]. The example of the post-DSO channel map in the UK is provided in Figure 1.

It is envisaged that cleared spectrum be allocated to specific and potentially licensed services. This is already the case of the $800 \mathrm{MHz}$ band (ch61-ch68), where a continuous portion of $72 \mathrm{MHz}$ has been harmonized across Europe, Middle East, and Africa [19] and in the UK [20] for potential cellular extensions, and further harmonization of the digital dividend is still to be expected [21]. Thus, CR technology is foreseen to occur in the interleaved spectrum where opportunistic usage is expected, as non-interference with incumbents is requested in these channels. Owing to the good propagation properties of UHF signals, various scenarios are foreseen [22].

In all countries where TVWS opportunistic access is allowed or considered, non-interference with incumbents relies on an overlay mechanism for which spectrum usage is allowed in channels vacant of any incumbent transmission [23]. Overlay mechanism implies that, before setting an opportunistic transmission, the opportunistic system ensures that the target band is vacant of incumbent operation. In fact, the actual constraint is that there should be no victim device (i.e., incumbent receiver) using the frequency targeted by the opportunistic system in the coverage area of this opportunistic system (Figure 2). The likelihood of interference is tied to the choice on parameter values, whether it is sensor sensitivity (when a sensing based approach is used), or positioning accuracy (when the "no talk area" is determined by a geo-location approach), and of course, emission power levels [24]. In any case, incumbent protection has to be traded against the inhibition of opportunistic access.

Although cognitive access is being considered by many regulators around the world, few of them have agreed on parameter figures. As far as spectrum sensing is concerned, the UK communications regulator (OFCOM) recommends a sensing sensitivity of $-120 \mathrm{dBm}$, assuming DTT receiver sensitivity of $-78 \mathrm{dBm}$ [11]. In its first set of rules, the US federal communications commission (FCC) recommended a DTT signal sensing sensitivity of $-114 \mathrm{dBm}$ [9]. It shall be noted that the OFCOM and the FCC consider channels of 8 and $6 \mathrm{MHz}$, respectively. An opportunistic system is also requested to detect potential apparition of an incumbent signal and to evacuate the band whenever this situation occurs. The FCC [9] requires that in-service monitoring is performed at least every $60 \mathrm{~s}$, and that the channel is released within an evacuation time of no more than $2 \mathrm{~s}$. The OFCOM considers more conservative figures, since time between sensing is limited to $1 \mathrm{~s}$ [11].

Similarly, transmit power of opportunistic systems is limited to levels that ensure non-interference with DTT systems. Again, these levels depend on the assumption

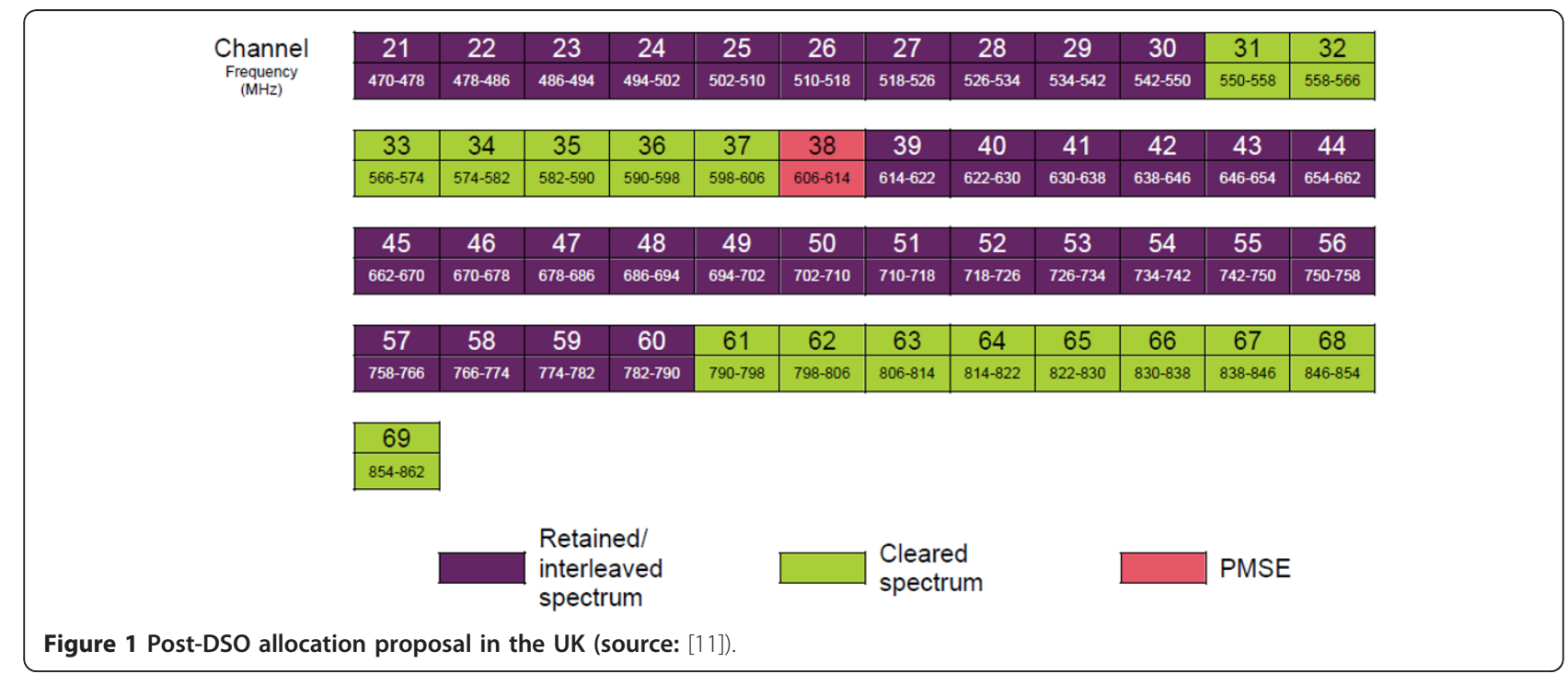




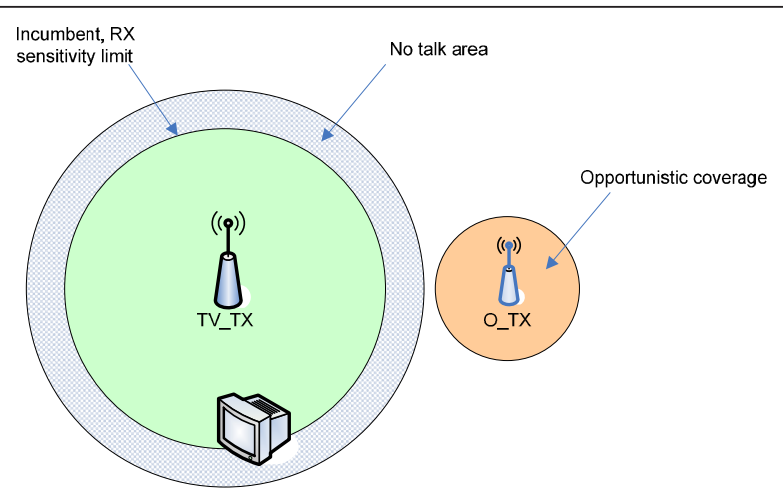

Figure 2 Example of TV (TV_TX) and opportunistic system $\left(O_{-} T X\right)$ operating in the same channel.

about the configuration of opportunistic transmitter to the victim device receiver (including geometry and antenna pattern). Altogether, [11] considers a path loss of $55 \mathrm{~dB}$ from the opportunistic transmission to the victim device. Then, taking into account the rejection capabilities of TV channelization filters (a cautious value is typically $30 \mathrm{~dB}$ ), and a safety margin of $3 \mathrm{~dB}$, it can be concluded that opportunistic system maximal power level should be set to $4 \mathrm{dBm}(2.5 \mathrm{~mW})$ equivalent isotropically radiated power (EIRP) in channels adjacent to DTT services. However, in non-adjacent channels, filters have much better rejection capabilities, and the OFCOM concluded that $17 \mathrm{dBm}(50 \mathrm{~mW})$ EIRP could be allowed in these channels. Similarly, out-of-band radiation has to be limited considering the $-78 \mathrm{dBm}$ sensitivity and a $20 \mathrm{~dB}$ co-channel carrier over interference $(C / I)$ ratio. Considering the same path loss and a safety margin as above, [11] concludes that out-of-band radiation should be kept below $-46 \mathrm{dBm}$. This results in spectrum masks presented in Figure 3.

The FCC defines power levels in a different way. First, two types of devices are considered in [9]. Fixed devices (FDs) are allowed to operate at up to $30 \mathrm{dBm}(1 \mathrm{~W})$ transmit power and with a gain antenna to achieve $36 \mathrm{dBm}(4$ W) EIRP. Personal/portable devices (PPD) are allowed to operate at up to $20 \mathrm{dBm}(100 \mathrm{~mW})$ EIRP in non-adjacent channels (with PSD of $1.67 \mathrm{~mW} / 100 \mathrm{kHz}$ ). This limit is reduced to $16 \mathrm{dBm}(40 \mathrm{~mW})$ EIRP when operating on a channel adjacent to a TV-occupied channel (with PSD of $0.7 \mathrm{~mW} / 100 \mathrm{kHz}$ ). Antenna gain is not allowed for PPD. The FCC also modifies the power requirements of PPDs according to the way primary systems are detected. The FCC suggests that PPDs should either have geo-location capabilities or be under the control of an FD. The FCC imposes PPD that do not operate under these conditions to have spectrum sensing capabilities to detect TV stations. However, transmit power is limited to $17 \mathrm{dBm}(50$ $\mathrm{mW}$ ) EIRP for these devices. Other than transmit powers, the FCC states that out-of-band emissions should be limited to $55 \mathrm{~dB}$ below the power level in the bands they occupy, as measured in a band of $100 \mathrm{kHz}$. It should be noted that FDs are not allowed to operate in adjacent channels, which means that the maximal interference level should be calculated considering the $4 \mathrm{~mW}$ condition, leading to levels similar to the one of the OFCOM (-47 $\mathrm{dBm}$ for a $6 \mathrm{MHz}$ channel). Table 1 recaps the key parameters as required by the FCC and OFCOM that will be further analyzed in the rest of the article.

\section{Spectrum vacancy detection}

The main purpose of vacant channel detection is to protect TV bands incumbents: DTT and program making

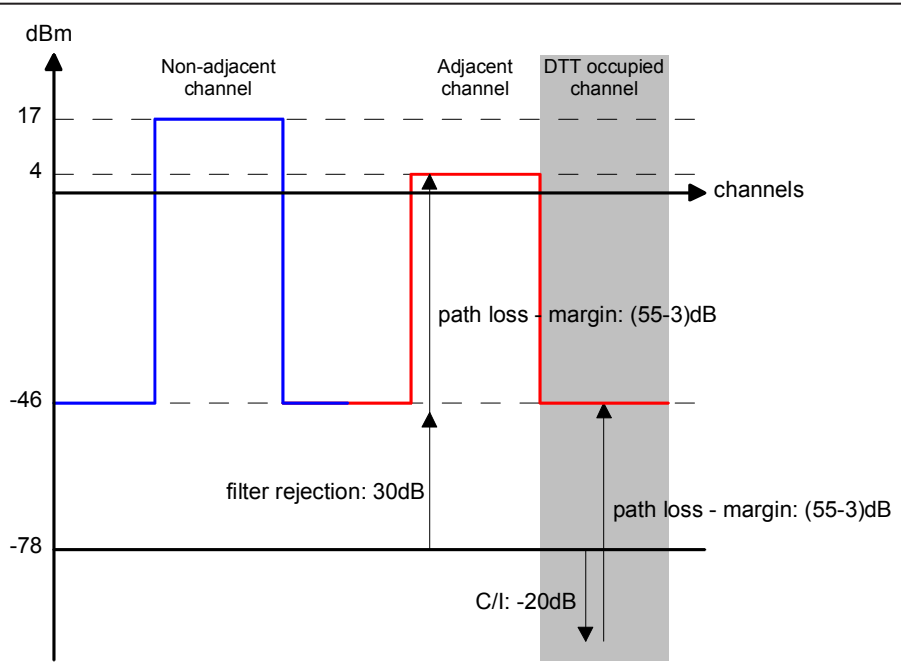

Figure 3 Spectrum mask for opportunistic usage in the TVWS as suggested by OFCOM. 
Table 1 Key FCC and OFCOM requirements

\begin{tabular}{|c|c|c|c|}
\hline Parameter & FCC [9] & OFCOM [11] & Note \\
\hline Sensing sensitivity for DTT & $-114 \mathrm{dBm}$ & $-120 \mathrm{dBm}$ & \\
\hline Sensing sensitivity for wireless microphones & $-114 \mathrm{dBm}$ & $-126 \mathrm{dBm}$ & In $200 \mathrm{kHz}$ bandwidth \\
\hline Power for FD in adjacent band & $\begin{array}{l}\text { Not } \\
\text { allowed }\end{array}$ & $\begin{array}{l}\text { Not } \\
\text { applicable }\end{array}$ & \\
\hline Power for FD in non-adjacent band with geo-location capability & $30 \mathrm{dBm}$ & $\begin{array}{l}\text { Not } \\
\text { applicable }\end{array}$ & FCC: $36 \mathrm{dBm}$ EIRP with a gain antenna \\
\hline Power for PPD in adjacent band & $16 \mathrm{dBm}$ & $4 \mathrm{dBm}$ & Gain antenna not allowed \\
\hline Power for PPD in non-adjacent band with geo-location capability & $20 \mathrm{dBm}$ & $17 \mathrm{dBm}$ & Gain antenna not allowed \\
\hline $\begin{array}{l}\text { Power for PPD in non-adjacent band without geo-location } \\
\text { capability }\end{array}$ & $17 \mathrm{dBm}$ & & \\
\hline Out-of-band performance & $<55 \mathrm{~dB}$ & $<-46 \mathrm{dBm}$ & $\begin{array}{l}\text { Relative to in-band power in the case of the } \\
\text { FCC }\end{array}$ \\
\hline In-service monitoring period & $60 \mathrm{~s}$ & $1 \mathrm{~s}$ & \\
\hline Evacuation time & $2 \mathrm{~s}$ & Not specified & \\
\hline
\end{tabular}

and special events (PMSE) services. Different approaches have been envisaged by regulators to determine whether spectrum is free: spectrum sensing, geo-location, and beaconing, although the latter has not been identified as a suitable option $[11,25]$.

WSD is based on sensing assess spectrum vacancy without any support from the infrastructure. The hidden device problem may result in some residual probability of interference [26]. This has pushed regulators to consider very conservative scenarios and to impose very high sensitivity requirements (see Table 1). Moreover, signals in adjacent channels and fluctuating signal levels may lead to even more demanding detection contexts. Several blind and signal-specific feature-based techniques have been proposed to perform sensing: spectral correlation, time-domain cyclostationarity, eigenvalue decomposition, pilot detection, higher order statistics analysis [27].

In the UHF TV bands (typically 470-862 MHz), the primary system is the DTT broadcast. Thus, the detector can exploit a priori knowledge of the waveform to improve its sensitivity at low signal-to-noise ratio (SNR) [27]. In this article, DTT systems comply with the DVB$T$ standard. DVB-T is used in many countries including Europe, India, Australia, Russia, and some Asian, African and American countries. The DVB-T signal is built on the orthogonal frequency division multiplexing (OFDM) modulation. OFDM has strong cyclostationary features stemming from the presence of the cyclic prefix (CP). For this reason, cyclostationarity detector has been proposed as one of the most promising technique for DVB-T signal sensing [28]. Theoretical aspects of cyclostationary detection have been thoroughly addressed in the literature [29-32], and more recently in the specific context of CR [28,33-36]. Implementation of cyclostationary spectrum density (CSD) detectors has been proposed in $[37,38]$ in a general CR context where the cyclic frequencies of the signal to detect are unknown. In [39], we proposed a hardware architecture for specific OFDM waveforms (i.e., WiFi and DVB-T) based on the cyclostationarity algorithm presented in [28]. It performs like CSD where the analyzed cyclic frequencies are restricted to the ones of the a-prioriknown waveform. As [39] is tailored to these specific cyclic tones, the implementation complexity could be reduced compared with CSD. It was also highlighted in [39] that the DVB-T detector can benefit from long integration time thanks to the broadcast nature of the DTT signal.

In order to assess the performance of [39] against the specifications of Table 1, the DVB-T detector was implemented on a complete testbed including an RF front-end [40]. The DVB-T standard defines different fast Fourier transform (FFT) sizes $(2048,4096$, or 8192) and different CP lengths (1/32, 1/16, 1/8, 1/4). In practice, deployments consider a smaller set of parameters depending on the country. The proposed architecture is tuned to the DVB-T parameter set used in France $($ FFT size $=8192$ and $\mathrm{CP}$ ratio $=1 / 32$ ). Figure 4 gives the sensing sensitivity of the complete testbed as a function of the sensing duration, considering a probability of false alarm of $10 \%$ and a probability of detection of $90 \%$. It is compared to the sensitivity obtained by simulations under perfect channel conditions as in [28]. Simulated results are provided, which consider a perfect RF with a zero noise figure to show the detector's sensitivity bound. They show that the $-120 \mathrm{dBm}$ sensitivity could be reached after $150 \mathrm{~ms}$. The gap between simulation and measurements is approximately $10 \mathrm{~dB}$ and comes from implementation issues as discussed in [40]. Because the front-end operates close to its sensitivity limit, the main contributor to the measurement degradation is the $9 \mathrm{~dB}$ noise figure of the front-end. 


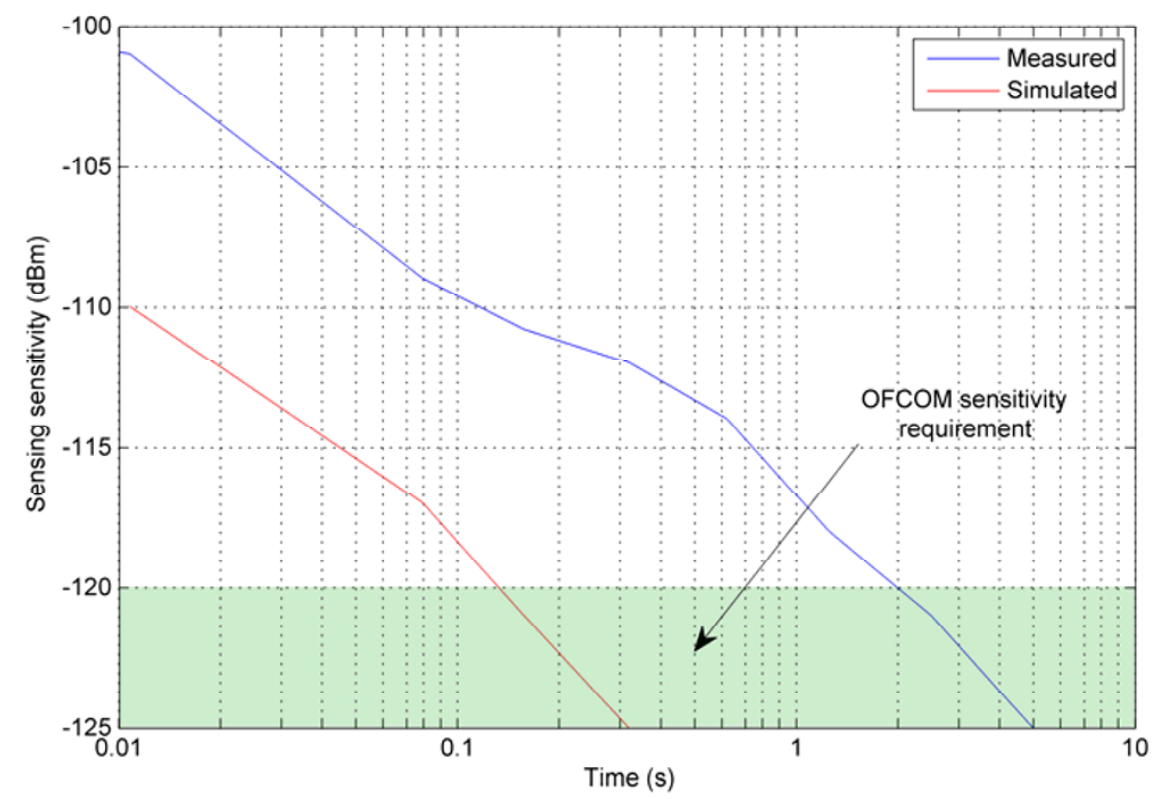

Figure 4 Sensitivity versus sensing duration of the cyclostationnarity detector.

Consequently, measurement results show that at least 2 seconds are needed to reach the sensing sensitivity required by OFCOM's recommendation. Thus, the proposed architecture fits the relaxed requirements of the FCC but needs to be improved to reach the OFCOM ones (see Section 2). Moreover, it is expected that integration time will be larger than channel coherence time $\left(T_{\mathrm{c}}\right)$, even for the simulated case (e.g., $T_{\mathrm{c}}=65 \mathrm{~ms}$, at $700 \mathrm{MHz}$ and for mobile scenario of $10 \mathrm{~km} / \mathrm{h}$ according to [41]). Therefore, additional channel variation compensation would be required, leading to a more complex implementation.

Furthermore, performance results of Figure 4 only consider one set of DVB-T parameters, which is a favorable scenario. In some countries, DVB-T broadcast may use several parameter sets and the sensor must be able to detect each of these configurations. Scanning several parameter sets would increase the complexity of the detector or would result in longer sensing time.

The main PMSE services to be detected are wireless microphones. Most of the literature references suggest the use of blind detection [42]. They are based on eigenvalue decomposition, spectral correlation, or energy detection [26]. Wireless microphone FM signal occupies a $200 \mathrm{kHz}$ band in a $8 \mathrm{MHz}$ channel. In [43], we propose a semi-blind detector based on the Teager-Kaiser energy operator [44]. It is based on the nonlinear model of the FM signal energy to estimate the energy accurately. An alternative promising solution performs energy calculation in the frequency domain on the microphone actual bandwidth [45]. It uses an FFT to compute the frequency response of the signal. Once the FFT is performed, the $8 \mathrm{MHz}$ band can be decomposed into sub-bands, and the energy can be estimated on each sub-band. This algorithm is called the "frequency domain energy detector."

The Teager-Kaiser energy detector allows a $2 \mathrm{~dB}$ detection gain compared to the energy detection, while the frequency domain energy detector allows a $5.5 \mathrm{~dB}$ detection gain and outperforms the autocorrelation detection as discussed in [43].

The choice of detector is also a trade-off between its performance and its complexity. Table 2 gives the complexity of each detector in terms of number of multiplications. The Teager-Kaiser energy detector does not induce an important increase in the computational complexity compared to the energy detector, while the frequency-domain energy detector and the autocorrelation detector have a much more important complexity.

How these detectors meet the regulation requirements presented in Section 2 is the main objective of our analysis. Figure 5 gives simulated sensing sensitivity as a function of the sensing duration for each detector. The sensitivity requirement in [11] is quoted for $200-\mathrm{kHz}$ channels. Thus, in order to process a complete $8 \mathrm{MHz}$ channel, either the same algorithm is applied to the 8 $\mathrm{MHz}$ channel, or successively to subchannels of 200 $\mathrm{kHz}$. In the first case, sensitivity is reduced by $16 \mathrm{~dB}$. In the latter case, the detection duration should be multiplied by the number of $200 \mathrm{kHz}$ steps within the $8 \mathrm{MHz}$ channel. This would end up to $480 \mathrm{~ms}$ for the frequency-domain energy detector if steps of $100 \mathrm{kHz}$ were 
Table 2 Complexity evaluation of the wireless microphone detectors for Ns samples

\begin{tabular}{ll}
\hline & Complexity in number of complex multiplication \\
\hline Energy detector & $\mathrm{Ns}$ \\
Teager-Kaiser detector & $2^{*} \mathrm{Ns}$ \\
Autocorrelation detector & $\mathrm{Ns} s^{*} \mathrm{Ns}$ \\
Frequency domain energy detector & $\mathrm{Ns}^{*} \log _{2}(\mathrm{Ns})+\mathrm{Ns}$ \\
\hline
\end{tabular}

considered. The detection duration reaches $2400 \mathrm{~ms}$ in the case of the Teager-Kaiser energy detector. These results are reached under perfect channel conditions without fading and radio or hardware impairments. As we concluded for the DVB-T signal detection, this performance is expected to be degraded in a real system with hardware implementation of the algorithms, where limitations from front-end noise figure has to be introduced.

From these results, it can be understood that sensing is not foreseen as a short term large market option considering current requirements. The issue is made even more complex in the case of PMSE services which are more dynamic than DTT transmitters. One option is to have a harbor channel for PMSE transmission and to prohibit the opportunistic use of this channel. This solution has been adopted in the UK where channel 38 is already dedicated to PMSE transmission (see Section 2). However, in many countries, PMSE services are allowed to use any of the UHF bands, and this solution will induce deep modifications for the PMSE manufacturers, making it hard to generalize to all countries.
Geo-location-based approach is an alternative to sensing. The FFC and the OFCOM recommend location accuracies of $50 \mathrm{~m}$ [9] and $100 \mathrm{~m}$ [11], respectively. The accuracy of commercially available global positioning system (GPS) receivers meets these requirements [46] where the signal is available. Hybrid geo-location technologies have emerged to extend GPS coverage [46,47] (e.g., for indoor applications). Therefore, providing geolocation information is not a major challenge for a WSD. Channel vacancy assessment requires a global infrastructure where the location information is an entry to a database. This centralized approach enables a more flexible management, since the protection margins may be globally modified by updating the database. The main challenges are on the database management, maintenance, and information exchange. They have been identified by the OFCOM [48]. For instance, how frequently should the database be updated is an open issue to consider non-permanent incumbents, such as PMSE devices. Another issue is the propagation model to be considered to determine white space areas. Accurate models need to be developed, as conservative ones

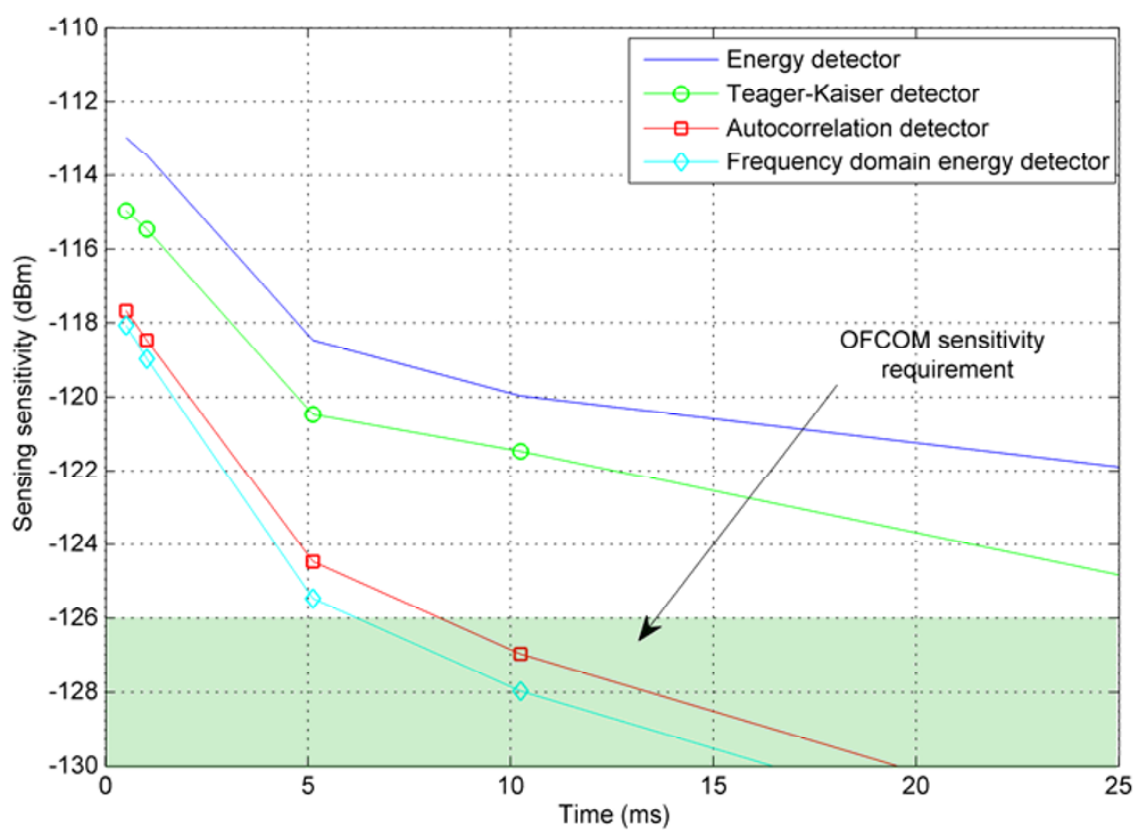

Figure 5 Sensing sensitivity versus sensing duration of several wireless microphone detectors. 
would lead to a dramatic reduction of available white space.

\section{Modulation schemes for opportunistic radio}

Multicarrier modulation techniques in general, and OFDM in particular, have developed as the most adapted techniques for broadband wireless communications. These waveforms have proven their worth in various applications and standards, and are commonly deployed in broadcast applications (DAB, DVB-T) as well as mobile wireless communications (WLAN, LTE). A relatively simple equalization and high bandwidth efficiency have been so far the main motivations for using OFDM when data are to be transmitted over a wireless multipath channel. Owing to the narrowband subcarriers, the CP in combination with channel coding, OFDM modulation techniques have led to very robust and reliable systems. The modulation technique is therefore a natural choice for CR in the TVWS.

However, when it comes to spectral properties, OFDM waveform shows some limitations. An analytic expression for the power spectral density of the OFDM with CP (CP-OFDM) waveform is proposed in [49]. The result of the derived expression is recalled in (1):

$$
P(f)=\frac{1}{T_{s}} \sum_{k}\left|G_{k}(f)\right|^{2}\left[1+2 \frac{C P}{N+C P} \cos \left(\frac{2 \pi f}{\Delta f}\right)\right]
$$

where $N$ is the number of carriers in the OFDM waveform, and $\mathrm{CP}$ is the duration of the cyclic prefix, $\Delta$, is the carrier spacing, and $G_{k}(f)$ is the power spectral density of the pulse on carrier $k$. Assuming a brickwall filter, $G_{k}(f)$ becomes the $\sin c^{2}$ function centered on the active carrier. Figure 6 describes the power spectral density function of an OFDM waveform exhibiting similar parameters as defined in LTE: carrier spacing is set to $15 \mathrm{kHz}$, the size of FFT is set to 1024 , and CP to 72 samples. Different active bandwidths corresponding to different number of active carriers are represented assuming an $8 \mathrm{MHz}$ emission spectrum mask (ESM).

The results highlight the necessity for a transmit filter cascaded after the modulator to limit the leakage of outof-band signal power and meet the rejection level requirements of $55 \mathrm{~dB}$ introduced in Section 2. In order to understand the requirements on the transmit filter, we derive the rejection requirement on the transmit filter and then extrapolate the number of taps required for a finite impulse response implementation of the filter. The filter length is estimated using an equiripple lowpass implementation and should be applied to both real and imaginary components of the baseband signal. In order to keep the distortion of the OFDM signal to a reasonable level at the output of the transmitter, the ripple in the band is set to $0.5 \mathrm{~dB}$, while the end of the passband is set to the limit of the occupied bandwidth.

Table 3 summarizes the results for the different scenarios of Figure 6.

The length and the complexity of the transmit filter increase dramatically as the guard band is reduced. A compromise between spectral efficiency and complexity has to be found.

The main shortcoming of the OFDM waveform identified here originates from the large side-lobes because of the rectangular shaping of the temporal signal. Severe out-of-band leakage constraints have been set to guarantee non-interference to adjacent incumbent transmission. Therefore, OFDM-based opportunistic radio

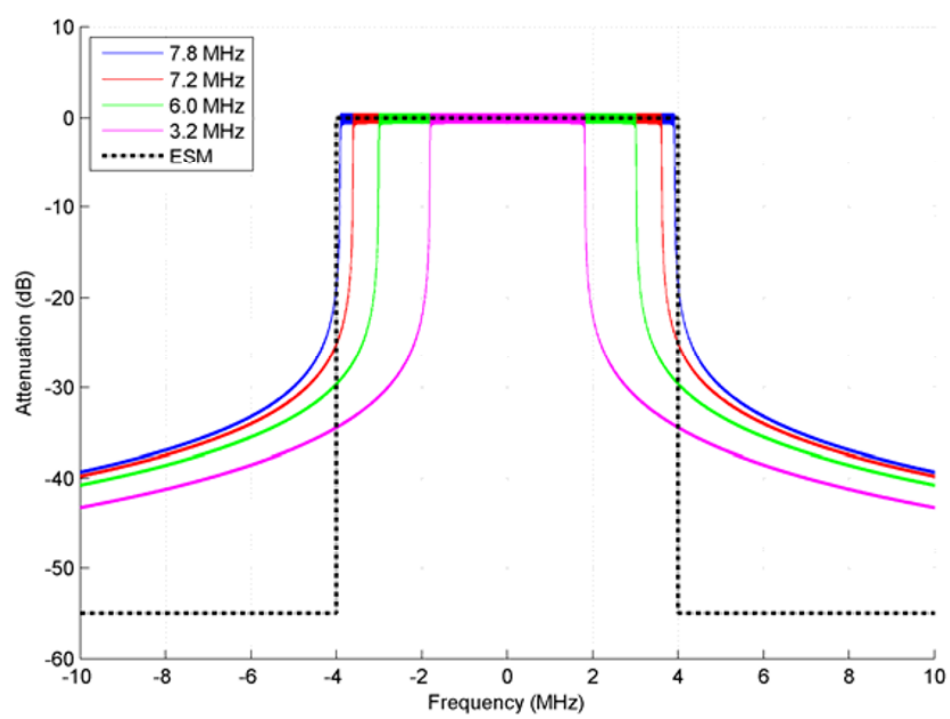

Figure 6 Power Spectral Density of CP-OFDM waveforms 
Table 3 Complexity of transmit filter as a function of occupied bandwidth

\begin{tabular}{lllllr}
\hline $\begin{array}{l}\text { Carrier } \\
\text { spacing }\end{array}$ & $\begin{array}{l}\text { Occupied } \\
\text { bandwidth }(\mathbf{M H z})\end{array}$ & $\begin{array}{l}\text { Number of active } \\
\text { carriers }\end{array}$ & $\begin{array}{l}\text { Guard } \\
\text { bandwidth }(\mathbf{M H z})\end{array}$ & $\begin{array}{l}\text { Requirement on Tx filter rejection to meet } \\
\mathbf{5 5} \text { dB rejection } \\
\mathbf{( d B})\end{array}$ & $\begin{array}{l}\text { Length of baseband } \\
\text { Tx filter }\end{array}$ \\
\hline $15 \mathrm{kHz}$ & 7.8 & 520 & 0.1 & 35.4 & 230 \\
& 7.2 & 480 & 0.4 & 30.5 & 52 \\
& 6.0 & 400 & 1.0 & 25.4 & 18 \\
& 3.2 & 240 & 2.2 & 20.4 & 6 \\
\hline
\end{tabular}

transmitters require either very complex transmit filters, or large frequency guard. A solution introduced by Chang [50] and Saltzberg [51] in the mid-1960s suggests combining filtering and multicarrier modulation techniques. This solution is becoming a strong contender to replace OFDM for CR applications [52], whenever drastic adjacent incumbent protection is required, as in the case of TVWS communication. This alternative solution is known as filter bank multicarrier communication techniques or FBMC.

FBMC allows the control of the frequency response of each carrier by introducing a filter bank, $g_{k}$, centered on every active carrier and based on the same prototype response. The orthogonality of overlapping carriers is not preserved as for OFDM techniques, and the symbol spans over more than one symbol duration. Instead, an offset-QAM (OQAM) transmitted at twice the symbol rate enables mitigation of the impact of intersymbol and intercarrier interference, while keeping the same overall bandwidth [53]. This technique is usually referred to as OFDM/OQAM in the literature.

Figure 7 compares OFDM and FBMC spectra using a prototype filter as introduced in [54]. The proposed prototype filter spans over $K$ OFDM symbols. $K$ is selected as a trade-off between adjacent carrier power rejection and complexity. A conservative value of $K=4$ has been chosen to meet the rejection requirement level of $55 \mathrm{~dB}$. The power spectral density of the FBMC signal can be expressed as in (1) where the CP is set to 0 , and the $G_{k}$ (f) corresponds to the prototype filter.

The FBMC waveform does not require any external transmit filter to meet the spectral constraints imposed on the adjacent channel spectrum leakage, while as already underlined earlier CP-OFDM requires a transmit lowpass filter of around 52 taps applied on both real and imaginary parts of the signal assuming an occupied bandwidth of $7.2 \mathrm{MHz}$.

OFDM/OQAM structure is based on the transmultiplexer described in Figure 8 and consists in a serial-toparallel conversion, an OQAM pre-processing, and synthesis filters. An analysis filter followed by the OQAM post-processing completes the receiver. Hirosaki [55] has shown that the structure may be efficiently implemented using an inverse FFT, followed by a polyphase network (PPN) structure as illustrated in Figure $8 b$.

The complexity of FBMC can then be evaluated and compared with CP-OFDM. A simple way to estimate complexity is to evaluate the total number of real multiplications for both options. Assuming split-radix implementation of the IFFT and FFT, the number of real multiplications per multicarrier symbol is given in [56]

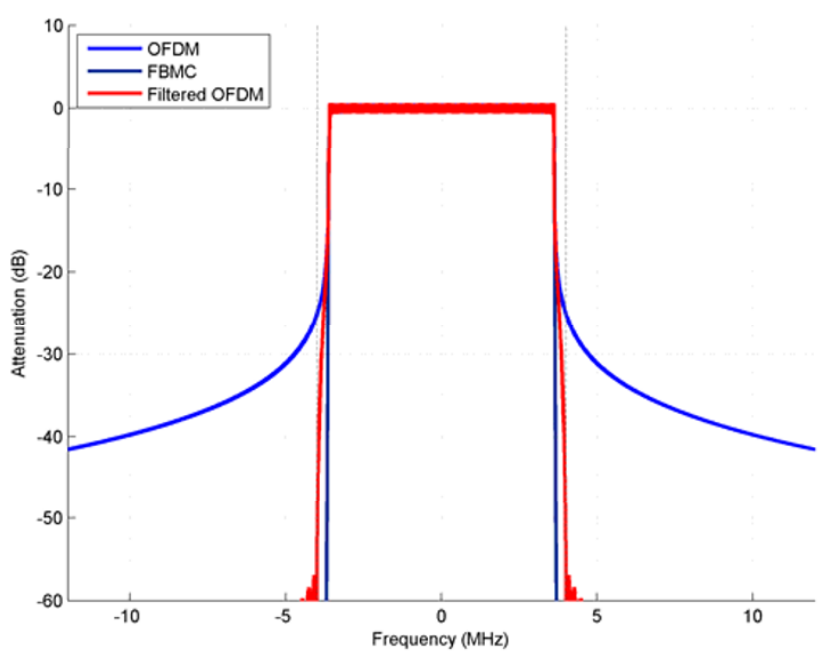

Figure 7 Power Spectral Density-CP-OFDM versus FBMC (7.2-MHz occupied bandwidth). 


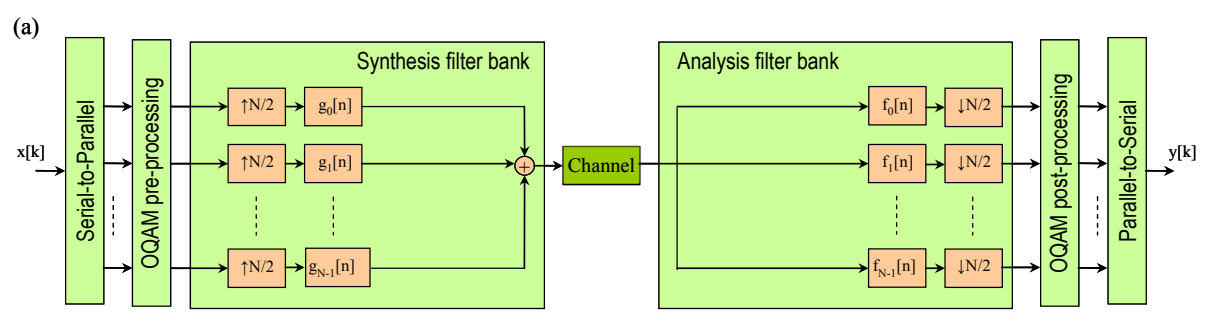

(b)

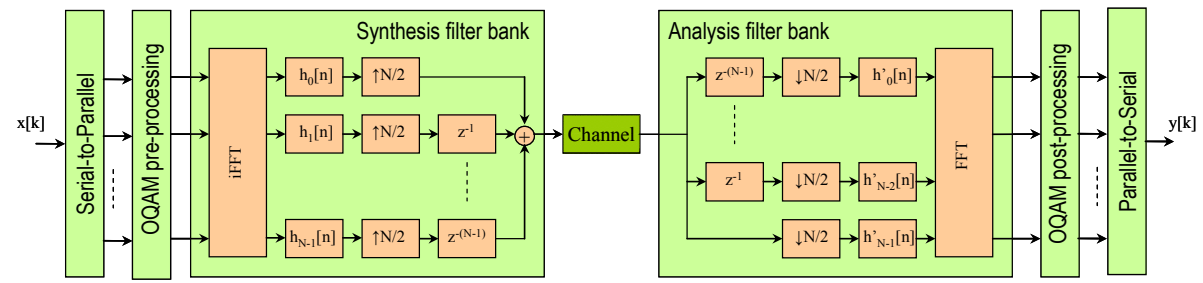

Figure 8 Modulation/Demodulation of OFDM/OQAM. (a) direct structure. (b) FFT and PPN.

for the FBMC modulator and demodulator. For OFDM, the complexity is determined by the IFFT and FFT of size $N$.

$$
\begin{aligned}
C_{F B M C} & =4\left(2 N+\left(N\left(\log _{2}(N)-3\right)+4\right)+2 K N\right) \\
C_{C P-O F D M} & =2\left(N\left(\log _{2}(N)-3\right)+4\right),
\end{aligned}
$$

In (2), $N$ is the number of carriers in the FFT, and the FBMC prototype filter spans over $K$ OFDM symbols. Equation (2) does not include the complexity of the transmit filter. Using the same parameters as used for building Table 4, we can compare the complexities of FBMC and CP-OFDM as a function of the occupied bandwidth.

As FBMC does not require any transmit filter, as its in-built filter matches adjacent channel requirement; its complexity does not change with the occupied bandwidth. For CP-OFDM, complexities of modulation and demodulation are heavily dependent on the length of the transmit filter. Values in Table 4 do not take complexity of the equalizer into account. When considered, complexity figures are mitigated, and CP-OFDM and FBMC are expected to be comparable.

Its ability to perform spectrum aggregation is another challenge a waveform adapted to the TVWS must face. Opportunistic users may get access to a spectrum that is already heavily fragmented. If the spectral needs are not met in a contiguous space of spectrum, then some form of aggregation should be realized. An efficient form of spectrum aggregation is the OFDM-based spectrum pooling as presented in [57]. The idea is to match the bandwidth of one sub-band with an integer multiple of the carrier spacing, $\Delta$, used by the opportunist transmitter.

An example of multicarrier-based spectrum pooling is given in Figure 9. Two key advantages have been identified for the transceiver architecture. First, the input of the IFFT can be fed with zeros at frequencies where incumbent users are present, while vacant subbands are used for transmission by the opportunistic radio. Second, the FFT operation required at the receiver to invert the modulation may also be used to monitor spectral activity of the other users at no extra cost. Both OFDM and FBMC may theoretically be suited to multicarrier-based spectrum pooling. However, in the TVWS context, the $55 \mathrm{~dB}$ adjacent channels' rejection cannot be met without a very complex and programmable band-pass transmit filter in the $\mathrm{CP}$ OFDM case, whereas FBMC would simply requires "switching on and off" the appropriate carriers at the transmitter.

Table 4 Complexity comparison in number of real multiplications per symbol $(N=1024, K=4)$

\begin{tabular}{llllll}
\hline $\begin{array}{l}\text { Carrier } \\
\text { spacing }\end{array}$ & $\begin{array}{l}\text { Occupied bandwidth } \\
(\mathbf{M H z})\end{array}$ & $\begin{array}{l}\text { Complexity CP- } \\
\text { OFDM }\end{array}$ & $\begin{array}{l}\text { Complexity of Tx filter for CP- } \\
\text { OFDM }\end{array}$ & $\begin{array}{l}\text { Total complexity CP- } \\
\text { OFDM }\end{array}$ & $\begin{array}{l}\text { Total complexity } \\
\text { FBMC }\end{array}$ \\
\hline $15 \mathrm{kHz}$ & 7.8 & 14344 & 471040 & 485384 & 69648 \\
& 7.2 & & 106496 & 120840 & \\
& 6.0 & & 36864 & 51208 \\
& 3.2 & & 12288 & 26632 \\
\hline
\end{tabular}




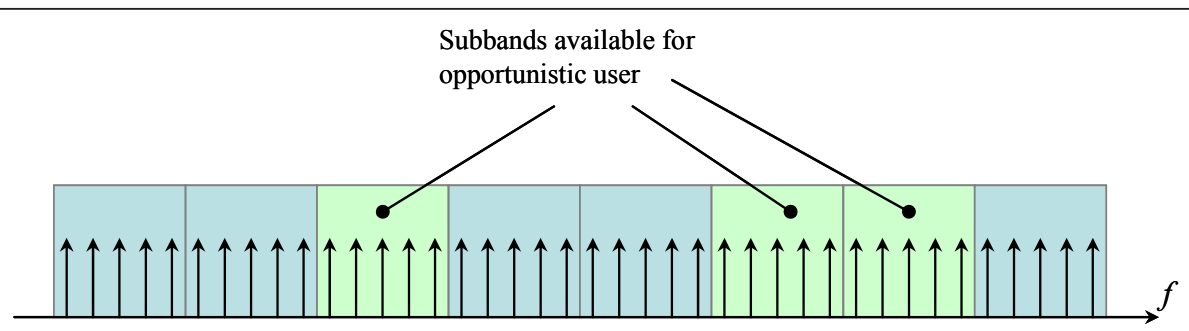

Figure 9 Example of multicarrier-based spectrum pooling.

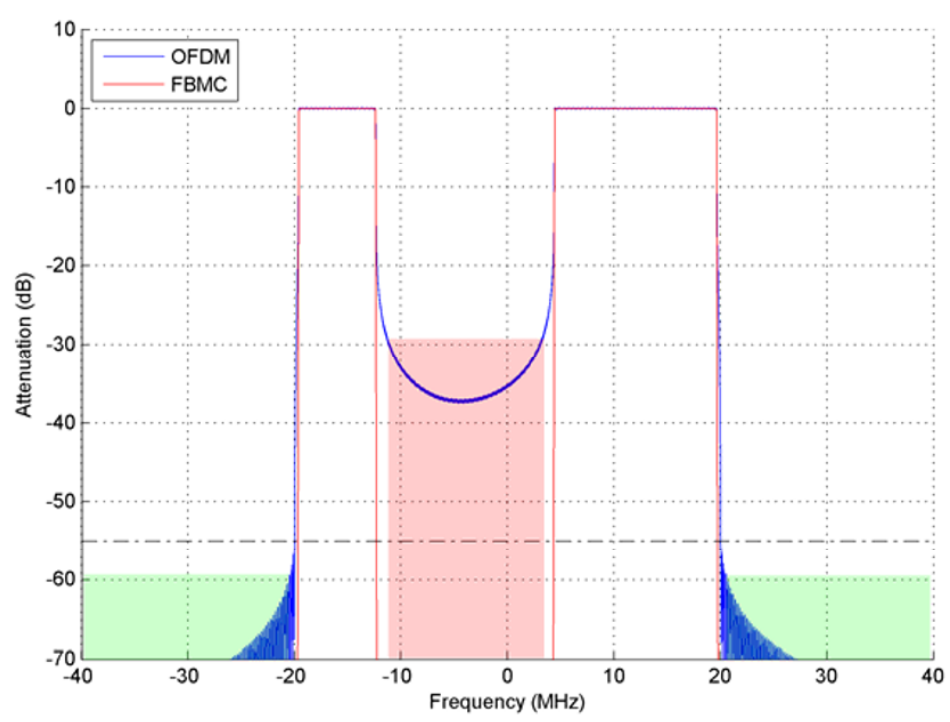

Figure 10 Multicarrier-based spectrum pooling, CP-OFDM versus FBMC

Figure 10 shows the CP-OFDM spectrum in the case of spectrum pooling where channels $[-20 \mathrm{MHz} ;-12$ $\mathrm{MHz}$ ] and [4 MHz; $20 \mathrm{MHz}$ ] are available for secondary transmission, and frequencies below $-20 \mathrm{MHz}$ and above $20 \mathrm{MHz}$, as well as [-12 $\mathrm{MHz} ; 4 \mathrm{MHz}]$ are allocated to incumbent system. The OFDM transmit filter rejects adjacent band leakage power on the spectrum below $-20 \mathrm{MHz}$ and above $20 \mathrm{MHz}$, but not in the [-12 $\mathrm{MHz} ; 4 \mathrm{MHz}$ ] band. On the other hand, the FBMC built-in filtering feature adapts to spectrum availability even in the fragmented case.

\section{Conclusion}

In this article, TVWS was discussed as a new and promising opportunity for CR. In these bands, some countries have assessed technical requirements for incumbent detection and opportunistic communication. Considering these requirements, technical options were reviewed and discussed. The access to interleaved spectrum requires spectrum vacancy assessment to insure that no harmful interference with incumbents is guaranteed. Significant study has been carried out in the field of spectrum sensing. This article showed that the regulator's requirements are difficult to achieve, specifically when sensing allowed time is limited. TV broadcast signals have features that can be exploited and the US regulation requirements can be met, when measurements can be performed over several seconds. This implies low mobility of the sensor. As far as wireless microphones are concerned, it was shown that detection is even more difficult because of the evanescence of the signal. Geo-location techniques translate the detection issue into a more complex global system architecture, which requires an up-to-date maintained, and reliable database, as well as means to insure that opportunistic radios can query the database. This requires another radio or a wired access to the database. Communication schemes for TVWS were also discussed. It was shown that despite its prominence in modern broadband radios, CP-OFDM has some drawbacks for TVWS operation. Indeed, CP-OFDM sidelobes make it inappropriate in adjacent channels, unless expensive 
rejection filters are used. Furthermore, new services claim for broadband access, which may be difficult to achieve in on TV channels of 6-8 MHz. Spectrum pooling is an appealing approach to virtually elaborate wider channels, but again CP-OFDM is not applicable because of unaffordable out-of-band leakage. FBMC was presented as a valid alternative to CP-OFDM, as it achieves both adjacent coexistence and spectrum pooling.

\section{Acknowledgements}

The research support leading to these results was derived from the European community's seventh framework program (FP7) under Grant Agreement number 248454 (QoSMOS).

\section{Competing interests}

The authors declare that they have no competing interests.

Received: 20 May 2011 Accepted: 15 November 2011

Published: 15 November 2011

\section{References}

1. J Mitola, Cognitive Radio: An Integrated Agent Architecture for Software Defined Radio (Ph.D. thesis, Royal Institute of Technology, Sweden, May 2000)

2. D Noguet, How to Reorganise Radio Spectrum Resources. eStrategies-Projects (British Publishers No 7, November 2008)

3. D Raychaudhuri, I Seskar, M Ott, S Ganu, K Ramachandran, H Kremo, R Siracusa, H Liu, M Singh, Overview of the ORBIT radio grid testbed for evaluation of next-generation wireless network protocols, in IEEE Wireless Communications and Networking Conference (WCNC) (2005)

4. SM Mishra, D Cabric, C Chang, D Willkomm, B van Schewick, A Wolisz, RW Brodersen, A real time cognitive radio testbed for physical and network level experiments, in IEEE International Symposium on New Frontiers in Dynamic Spectrum Access Networks (DYSPAN) (2005)

5. L Biard, D Noguet, T Gernandt, P Marques, AA Gameiro, Hardware demonstrator of an opportunistic radio system using temporal opportunities, in Cognitive Radio Oriented Wireless Networks and Communications (Crowncom), Hanover, Germany (2009)

6. TR Newman, D Depoy, T Bose, JH Reed, Designing and deploying a building-wide cognitive radio network testbed. IEEE Commun Mag. 48(9), 106-112 (2010)

7. WARP, Wireless open-Access Research Platform, Rice University. http://warp. rice.edu/

8. FCC Proposed Rule, Unlicensed Operation in the TV Broadcast Bands, US Federal Register 69(117), 34103-34111 (June 18 2004)

9. FCC Final Rule, Unlicensed Operation in the TV Broadcast Bands, US Federal Register 74(30), 7314-7332 (Feb 17 2009)

10. FCC Notice, Unlicensed Operation in the TV Broadcast Bands, US Federal Register 76(26), 6789-6792 (Feb 8 2011)

11. Digital Dividend, Cognitive Access, Statement on Licence-Exempting Cognitive Devices Using Interleaved Spectrum (OFCOM, July 2009)

12. CEPT, Report from CEPT to the European Commission in response to the Mandate on the identification of Common and Minimal (Least Restrictive) Technical Conditions for 790-862 MHz for the Digital Dividend in the European Union, CEPT Report 30 http://www.erodocdb.dk/Docs/doc98/official/pdf/ CEPTREP030.PDF (October 2009)

13. BP Freyens, M Loney, Opportunities for white space usage in Australia, in 2nd International Conference on Wireless Communications, Vehicular Technology, Information Theory and Aerospace in Electronic Systems Technology (Wireless VITAE) (Chennai, India, March 2011)

14. IEEE 80222, Wireless Regional Area Networks (WRANs), http://www.ieee802. org $/ 22 /$

15. IEEE 80211af, TV White Space Study Group AF, Wireless Local Area Network (WLAN), http://www.ieee802.org/11/

16. ECMA392 Standard, MAC and PHY for Operation in TV White Space, http:// www.ecma-international.org/publications/standards/Ecma-392.htm
17. J Wang, MS Song, S Santhiveeran, K Lim, G Ko, K Kim, SH Hwang, M Ghosh, $\checkmark$ Gaddam, K Challapali, First cognitive radio networking standard for personal/portable devices in TV white spaces, in IEEE International Symposium on New Frontiers in Dynamic Spectrum Access Networks (DYSPAN) (2010)

18. M Nekovee, Quantifying the availability of TV white spaces for cognitive access in the UK, in IEEE ICC, Dresden, Germany, (June 2009)

19. ITU, World Radio Conference 07. http://www.itu.int/TU-R/indexasp? category=conferences\&link=wrc\&lang=en

20. Digital Dividend, Clearing the $800 \mathrm{MHz}$ band, Statement, OFCOM (June 2009)

21. World Radiocommunication Conference Set for Early, http://www.tu.int/ net/itunews/issues/2009/08/36aspx (2012)

22. M Mueck, D Noguet, TV white space standardization and regulation in Europe, in 2nd International Conference on Wireless Communications, Vehicular Technology, Information Theory and Aerospace in Electronic Systems Technology (Wireless VITAE), Chennai, India (March 2011)

23. D Noguet, R Datta, PH Lehne, M Gautier, G Fettweis, TVWS regulation and QoSMOS requirements, in 2nd International Conference on Wireless Communications, Vehicular Technology, Information Theory and Aerospace in Electronic Systems Technology (Wireless VITAE), Chennai, India (March 2011)

24. M Mishra, A Sahai, How Much White Space is there?, Technical Report, Electrical Engineering and Computer Sciences, (University of California at Berkeley, January 2009)

25. ECC REPORT 159, Technical and Operational Requirements for the Possible Operation of Cognitive Radio Systems in the 'White Spaces' of the Frequency Band $470-790$ MHz, CEPT, Cardiff (January 2011)

26. D Cabric, A Tkachenko, R Brodersen, Spectrum sensing measurements of pilot, energy, and collaborative detection, in IEEE Military Communication Conference, 1-7 (October 2006)

27. D Noguet, YA Demessie, L Biard, A Bouzegzi, M Debbah, K Haghighi, P Jallon, M Laugeois, P Marques, M Muroni, J Palicot, C Sun, S Thilakawardana, A Yamaguchi, Sensing Techniques for Cognitive Radio-State of the art and trends http://dyspan-sc.org (April 2009)

28. P Jallon, An algorithm for detection of DVB-T signals based on their second-order statistics. EURASIP J Wireless Commun Netw, 1-9 (2008)

29. WA Gardner, Statistical Spectral Analysis: A Nonprobabilistic Theory (PrenticeHall, Englewood Cliffs, New Jersey, 1988)

30. WA Gardner, G Zivanovic, Degrees of cyclostationary and their application to signal detection and estimation. Sig Process. 22(3) (1991)

31. WA Gardner, M Spooner, Signal interception: performance advantages of cyclic-feature detectors. IEEE Trans Commun. 40(1), 149-159 (1992). doi:10.1109/26.126716

32. W Gardner, A William, Cyclostationarity in Communications and Signal Processing (IEEE Press, New York, 1994)

33. J Lunden, V Koivunen, A Huttunen, H Vincent Poor, Spectrum Sensing in Cognitive Radios Based on Multiple Cyclic Frequencies, http://arxiv.org/abs/ 07070909 (July 2007)

34. Z Ye, J Grosspietsch, G Memik, Spectrum Sensing Using Cyclostationary Spectrum Density for Cognitive Radios, in IEEE Workshop on Signal Processing Systems, Shanghai, China (Oct 17-19 2007)

35. M Ghozzi, M Dohler, F Marx, J Palicot, Cognitive radio: methods for detection of free bands. Elsevier Sci I Special Issue on Cognitive Radio, 794-805 (7 September 2006)

36. A Bouzegzi, P Jallon, P Ciblat, A second order statistics based algorithm for blind recognition of OFDM based systems, in GLOBECOM (2008)

37. V Turunen, M Kosunen, A Huttunen, S Kallioinen, P Ikonen, A Pärssinen, J Ryynänen, Implementation of cyclostationary feature detector for cognitive radios, in Proceedings of the 4th international conference cognitive radio oriented wireless networks and communications (CROWNCOM), Hannover, Germany (June 2009)

38. V Turunen, M Kosunen, S Kallioinen, A Pärssinen, J Ryynänen, Spectrum estimator and cyclostationary detector for cognitive radio, in European Conference on Circuit Theory and Design (ECCTD 2009), Antalya, Turkey, (August 23-27 2009)

39. D Noguet, L Biard, M Laugeois, Cyclostationarity detectors for cognitive radio: architectural tradeoffs. EURASIP J Wirel Commun Netw (2010)

40. M Gautier, M Laugeois, Ph Hostiou, Cyclostationarity detection of DVB-T Signal: testbed and measurement, in The First International Conference on Advances in Cognitive Radio (COCORA), Budapest, Hungary (April 2011)

41. Th S Rappaport, Wireless Communications: Principles and Practice (Prentice Hall, London, 1996) 
42. H-S Chen, W Gao, D Daut, Spectrum sensing for wireless microphone signals, in IEEE Sensor, Mesh and Ad Hoc Communications and Networks Workshops (SECON08) (June 2008)

43. M Gautier, M Laugeois, D Noguet, Teager-kaiser energy detector for narrowband wireless microphone spectrum sensing, in Proceedings of the 5th International Conference on Cognitive Radio Oriented Wireless Networks and Communications (CROWNCOM), Cannes, France, (June 2010)

44. JF Kaiser, On simple algorithm to calculate the energy of signal, in IEEE International Conference on Acoustics, Speech, and Signal Processing (ICASSP90) (April 1990)

45. NM Neihart, S Roy, DJ Allstot, A parallel multi-resolution sensing technique for multiple antenna cognitive radios, in IEEE International Symposium on Circuits and Systems (May 2007)

46. S Wang, J Ming, BK Yi, S Kwon, Location based services for mobiles: technologies and standards, in IEEE International Conference on Communications (ICC), Beijin, China, (2008)

47. H Liu, H Darabi, P Banerjee, J Liu, Survey of wireless indoor positioning techniques and systems, systems, man, and cybernetics, part c: applications and reviews. IEEE Trans. 37(6), 1067-1080 (2007)

48. Digital Dividend, Geolocation for Cognitive Access, OFCOM (November 2009)

49. T van Waterschoot, V Le Nir, J Duplicy, M Moonen, Analytical expressions for the power spectral density of CP-OFDM and ZP-OFDM signals. IEEE Sig Process Lett. 17(4), 371-374 (2010)

50. RW Chang, Synthesis of band-limited orthogonal signals for multichannel data transmission. Bell Syst Technol J. 45, 1175-1796 (1966)

51. BR Saltzberg, Performance of an efficient parallel data transmission system. IEEE Trans Commun Technol. COM-15, 805-813 (1967)

52. R Datta, M Gautier, V Berg, Y Futatsugi, M Ariyoshi, M Schühler, Zs Kollár, P Horváth, D Noguet, G Fettweis, Flexible Multicarrier PHY design for cognitive radio in white space, in Proceedings of the 6th International Conference on Cognitive Radio Oriented Wireless Networks and Communications (CROWNCOM), Osaka, Japan (June 2011)

53. P Siohan, C Siclet, N Lacaille, Analysis and design of OFDM/OQAM systems based on filterbank theory. IEEE Trans Sig Process. 50(5), 1170-1183 (2002). doi:10.1109/78.995073

54. MG Bellanger, Specification and design of a prototype filter for filter bank based multicarrier transmission, in Proceedings of International Conference on Acoustics, Speech and Signal Processing (ICASSP '01). 4, 2417-2420 (2001)

55. B Hirosaki, An orthogonally multiplexed QAM sytem using the discret fourier transform. IEEE Trans Commun. 29(7), 982-989 (1981). doi:10.1109/ TCOM.1981.1095093

56. T Ihalainen, A Viholainen, TH Stitz, M Renfors, Generation of filter bankbased multicarrier waveform using partial synthesis and time domain interpolation, circuits and systems i: regular papers. IEEE Trans. 57(7), 1767-1778 (2010)

57. TA Weiss, FK Jondral, Spectrum pooling: an innovative strategy for the enhancement of spectrum efficiency. IEEE Commun Mag. 42(3), S8-S14 (2004)

doi:10.1186/1687-1499-2011-170

Cite this article as: Noguet et al: Advances in opportunistic radio technologies for TVWS. EURASIP Journal on Wireless Communications and Networking 2011 2011:170.

\section{Submit your manuscript to a SpringerOpen ${ }^{\mathcal{O}}$ journal and benefit from:}

- Convenient online submission

- Rigorous peer review

- Immediate publication on acceptance

- Open access: articles freely available online

- High visibility within the field

- Retaining the copyright to your article

Submit your next manuscript at $\gg$ springeropen.com 